\title{
Un estudio randomizado de terapia de reemplazo de nicotina en parches en el embarazo
}

\author{
Coleman T, Cooper S, Thornton JG, Grainge MJ, Watts K, Britton J, Lewis S; \\ Smoking, Nicotine, and Pregnancy (SNAP) Trial Team. N Engl J Med 2012;366:808-18.
}

Análisis crítico: Jorge Carvajal C. $P h D^{1}$, Claudio Vera P-G. $M S c^{1,2}$

${ }^{1}$ Unidad de Medicina Materno-Fetal, División de Obstetricia y Ginecología. ${ }^{2}$ Unidad de Medicina Basada en Evidencia, Facultad de Medicina, Pontificia Universidad Católica de Chile.

\section{RESUMEN (1)}

Introducción: La terapia de reemplazo con nicotina es efectiva para dejar de fumar fuera del embarazo y su uso es ampliamente recomendado durante el embarazo. Investigamos la eficacia y seguridad de parches de nicotina durante el embarazo. Métodos: Reclutamos participantes de siete hospitales en Inglaterra con edades entre 16 y 50 años, con embarazos entre 12 y 24 semanas de gestación, y quienes fumaran cinco o más cigarrillos diarios. Los participantes recibieron apoyo conductual para dejar de fumar y fueron asignados al azar a 8 semanas de tratamiento con parches de nicotina activos (15 mg por 16 horas) o parches placebo. El resultado primario fue la abstinencia desde la fecha de dejar de fumar hasta el parto, validado mediante medición de monóxido de carbono exhalado o cotinina salival. La seguridad fue evaluada monitorizando resultados adversos del embarazo y parto. Resultados: De 1050 participantes, 521 fueron asignados al azar a terapia de reemplazo con nicotina y 529 a placebo (Tabla I). No hubo diferencias significativas en la tasa de abstinencia desde la fecha de suspensión de fumar hasta el parto entre los grupos de tratamiento con nicotina y el placebo $(9,4 \%$ y $7,6 \%$ respectivamente; OR no ajustado 1,26 ; IC $95 \% 0,82-1,96)$, aunque la tasa fue más alta al mes en el grupo de terapia con nicotina que en el grupo placebo $(21,3 \%$ vs $11,7 \%)$. El cumplimiento fue bajo, solo $7,2 \%$ de las mujeres asignadas a terapia de reemplazo con nicotina y $2,8 \%$ de los asignados a placebo usaron los parches por más de un mes. Las tasas de resultados adversos en el embarazo y parto fueron similares en los dos grupos. Conclusiones: Agregar parches de nicotina (15 mg por 16 horas) al apoyo conductual para dejar de fumar a mujeres que fuman durante el embarazo, no mejora significativamente la tasa de abstinencia de fumar hasta el parto o el riesgo de eventos adversos durante el embarazo o parto. Sin embargo, las bajas tasas de cumplimiento limitan substancialmente la evaluación de seguridad.

\section{ANÁLISIS DE LA INVESTIGACIÓN}

\section{A. RELEVANCIA CLINICA DE LA INVESTIGACION}

Se ha establecido que el hábito tabáquico durante el embarazo empeora el resultado perinatal, específicamente aumentado el riesgo de parto prematuro, aborto espontáneo, restricción de crecimiento, muerte súbita neonatal y trastornos conductuales y psiquiátricos a largo plazo (2). Incluso recientemente se ha demostrado la asociación entre el hábito tabáquico durante el embarazo y un mayor riesgo de malformaciones congénitas (3). Se comprende con facilidad, que es indispensable suspender el hábito tabáquico durante el embarazo. En efecto, se considera al cigarrillo como el factor de riesgo perinatal prevenible, de mayor importancia.

Las terapias conductuales y farmacológicas han sido ampliamente recomendadas para lograr 
Tabla I

RESULTADOS DE ABSTINENCIA DEL HÁBITO TABÁQUICO EN EL EMBARAZO

\begin{tabular}{|c|c|c|c|c|}
\hline Resultado & $\begin{array}{l}\text { Nicotina* } \\
(n=521)\end{array}$ & $\begin{array}{l}\text { Placebo* } \\
(\mathrm{n}=529)\end{array}$ & $\begin{array}{c}\text { OR } \\
(\text { IC } 95 \%)\end{array}$ & $\begin{array}{l}\text { OR ajustado } \\
\text { (IC 95\%) }\end{array}$ \\
\hline $\begin{array}{l}\text { Primario } \\
\text { Abstinencia desde el día en que dejan de } \\
\text { fumar hasta el parto; evaluado por auto- } \\
\text { reporte y comprobado por medición de } \\
\text { monóxido de carbono exhalado y cotinina } \\
\text { en la saliva }\end{array}$ & $49(9,4)$ & $40(7,6)$ & $1,26(0,82-1,96)$ & $1,27(0,82-1,98)$ \\
\hline $\begin{array}{l}\text { Secundarios } \\
\text { Abstinencia por un mes desde el día en } \\
\text { que dejan de fumar, solo autoreporte }\end{array}$ & $131(25,1)$ & $74(14,0)$ & $2,07(1,51-2,85)$ & $2,13(1,54-2,95)$ \\
\hline $\begin{array}{l}\text { Abstinencia por un mes desde el día en } \\
\text { que dejan de fumar, validado }\end{array}$ & $111(21,3)$ & $62(11,7)$ & $2,05(1,46-2,88)$ & $2,10(1,49-2,97)$ \\
\hline
\end{tabular}

${ }^{*}$ Resultados expresados como n (\%)

cesación del tabaquismo en la población general, específicamente, el uso de parches de nicotina ha demostrado su eficacia en población general (4). Sin embargo, la eficacia de los parches de nicotina no ha sido demostrada durante el embarazo como un fármaco útil para reducir el hábito tabáquico y mejorar el resultado perinatal. Pese a la carencia de estudios significativos que avalen su uso, los parches de nicotina son recomendados en guías clínicas para reducir el tabaquismo en embarazadas (5).

\section{B. EL ESTUDIO (1)}

Diseño: Estudio clínico controlado randomizado doble ciego en 7 centros en Inglaterra. Pacientes: 1050 mujeres entre 16 y 50 años, con embarazos entre 12 y 24 semanas de gestación, y quienes fumaran cinco o más cigarrillos diarios. Intervención: 521 mujeres fueron asignadas a tratamiento por 8 semanas con parches de nicotina (15 mg por 16 horas) asociados a terapia de apoyo conductual para dejar de fumar. Comparación: 529 mujeres fueron asignadas a tratamiento por 8 semanas con parches de placebo, asociados a terapia de apoyo conductual para dejar de fumar. Resultado primario: Cese del hábito tabáquico al momento del parto (abstinencia desde el día en que dejan de fumar hasta el parto) evaluado por autoreporte y comprobado por medición de monóxido de carbono exhalado y cotinina salival. Resultados secundarios: Miden resultado perinatal y materno, y otros resultados respecto del cese del tabaquismo, como la abstinencia al final del primer mes desde el inicio de la suspensión (por autoreporte o comproba- do por monóxido de carbono exhalado o cotinina salival). Resultados: No se observaron diferencias significativas en el resultado primario. Entre los resultados secundarios medidos, el único que mostró diferencia significativa fue una mayor proporción de abstinencia por un mes, en el grupo que usó parches activos de nicotina. No hubo diferencias en el resultado perinatal.

\section{ANALISIS CRÍTICO}

Validez interna: Estudio con bajo riesgo de sesgo que describe apropiadamente criterios de inclusión, randomización, ocultamiento de la secuencia, ciego, seguimiento y análisis (Tabla II).

Resultados: El estudio demuestra ausencia de beneficio del uso de parches de nicotina asociados a terapia conductual para dejar de fumar durante el embarazo.

Si bien el estudio intenta demostrar la seguridad de esta droga, el poder del estudio no fue calculado para medir este efecto, de modo que estos resultados no son confiables, más aún si se considera que la tasa de cumplimiento con la intervención fue muy baja. Del mismo modo, no es posible hacer un estudio de eficacia de la droga (menos del 10\% de las pacientes usaron la droga por tiempo prolongado). Comentario: Estudio de buen diseño y sin fuentes evidentes de sesgo, con apropiado poder estadístico, que no muestra efectos del uso de nicotina en parches, asociado a terapia conductual, como una estrategia efectiva para dejar de fumar durante el embarazo. El cese del hábito tabáquico permite evitar los efectos adversos del tabaco en el embarazo, 
Tabla II

PLANILLA DE ANÁLISIS

Randomizado

Ocultamiento de la secuencia de randomización

Grupos similares al inicio

Cálculo tamaño muestral

Ciego:

Pacientes

Tratantes

Recolectores de datos

Adjudicador de eventos

Analistas o investigadores

Análisis por intención de tratar

Seguimiento

Cumplimiento intervención

\section{$\mathrm{Si}$}

$\mathrm{Si}$, randomización centralizada, estratificada por centros, en bloques permutados de tamaño variable

$\mathrm{Si}$

$\mathrm{Si}$, apropiado y completado

$\mathrm{Si}$

$\mathrm{Si}$

No especificado

No especificado

No especificado

$\mathrm{Si}$

El riesgo de sesgo por falla de seguimiento es mínimo. Se completó el seguimiento en: $93,1 \%$ grupo nicotina y $93,8 \%$ grupo placebo.

Muy bajo, solo el $7,2 \%$ de las pacientes del grupo nicotina y $2,8 \%$ del grupo placebo usaron los parches por más de un mes. siendo este un factor corregible. Debemos hacer el mayor esfuerzo posible para evitar el uso del cigarrillo en las embarazadas. La presente investigación muestra ausencia del beneficio del uso de la nicotina, y resalta la importancia de la terapia de apoyo conductual.

En Chile, la incidencia del tabaquismo en embarazadas se encuentra alrededor del $28 \%$, y menos del $50 \%$ de ellas suspende el hábito por estar embarazada (6). Se requiere de una estrategia a nivel nacional para enfrentar este problema.

\section{REFERENCIAS}

1. Coleman T, Cooper S, Thornton JG, Grainge MJ, Watts K, Britton J, Lewis S; Smoking, Nicotine, and Pregnancy (SNAP) Trial Team. A randomized trial of nicotine-replacement therapy patches in pregnancy. $\mathrm{N}$ Engl J Med. 2012;366:808-18.

2. Shea AK, Steiner M. Cigarette smoking during pregnancy. Nicotine Tob Res 2008;10:267-78.

3. Hackshaw A, Rodeck C, Boniface S. Maternal smoking in pregnancy and birth defects: a systematic review based on 173.687 malformed cases and $11,7 \mathrm{mi}-$ Ilion controls. Hum Reprod Update 2011;17:589-604.

4. Aubin HJ, Karila L, Reynaud M. Pharmacotherapy for smoking cessation: present and future. Curr Pharm Des 2011;17:1343-50.

5. Society for the Study of Addiction, treattobacco.net. Treatment guidelines (http://www.treatobacco.net/en/ page_224.php).

6. Mallol J, Brandenburg D, Madrid R, Sempertegui F, Ramírez L, Jorquera D. Prevalencia de tabaquismo durante el embarazo en mujeres chilenas de bajo nivel socioeconómico. Rev Chil Enferm Respir 2007;23:1722. 